\title{
Coherent Plasma Processes in Active Galactic Nuclei
}

\author{
V. Krishan \\ Instituto Nacional de Pesquisas Espaciais - INPE, \\ C.P. 515, 12201-970, São José dos Campos, SP, Brazil \\ and Indian Institute of Astrophysics Bangalore, 560 034, India
}

Received on 31 May, 2002

\begin{abstract}
The physics of active galactic nuclei (AGN) and related objects is one of the most actively pursued areas of astrophysics. Their large luminosities over the entire electromagnetic spectrum combined with extremely short time variability has stimulated a large number of astrophysicists to propose rather unconventional ideas. A variety of radiation mechanisms such as, thermal, synchrotron and Compton processes have been proposed to account for the complex continuum emission. It is only recently that the role of plasma radiation mechanisms has been shown to be of the utmost importance. It is demonstrated how a combination of the stimulated Raman and Compton scattering processes accounts for the major part of the spectrum, taking $3 \mathrm{C} 273$ as an example. In addition plasma mechanisms for, the heating of the emission line regions, absorption of $21 \mathrm{~cm}$ radiation, producing time variability on different time scales and pair production and annihilation are also discussed.
\end{abstract}

\section{Introduction}

Quasars are the most distant and the most luminous objects in the universe. The optical spectra of quasars consist of strong and broad emission lines, some of which have been identified to be the Balmer series of the hydrogen spectrum, shifted to longer wavelengths. The amount of shift implies that many of these objects are moving away at a large fraction of the speed $(c)$ of light. If this is a cosmological red shift then one finds using the Hubble law of the expansion of the universe, that these objects are at the farthest reaches of the universe. Quasars radiate almost over the entire electromagnetic spectrum with a typical power of $\sim 10^{46-47}$ erg $\mathrm{sec}^{-1}$. The sun, for example emits $\sim 3.9 \times 10^{33}$ erg $\mathrm{sec}^{-1}$. A typical galaxy has about $10^{11}$ stars in it. Thus the output of a quasars is much more than that of an entire galaxy. However, on optical images, it appears more like a star. Here lies the origin of the name Quasi-Stellar objects. Further, the observed rapid time $\left(t_{\nu}\right)$ variability of the radiation constrains the size $(d)$ of the source to be extremely small $\left(d<c t_{\nu}\right.$ if internal relativistic motions are ignored) typically of the order of $10^{14}-10^{15} \mathrm{~cm}$, comparable to the size of the solar system (Mushotzky, Done and Pounds, 1993).

What could be the nature of this 'little big' powerhouse? We know that the stars shine due to the thermonuclear burning of hydrogen and other elements. We are also aware of the efficiency $(\sim 0.007)$ with which mass is converted into radiation in a thermonuclear re- action. It was concluded that quasars need a mechanism more efficient than the thermonuclear burning. The consensus at present is that the energy extracted from the gravitational in-fall of gas on to a compact object such as a black hole (Rees, 1977), fits the bill. It is akin to terrestrial hydroelectric power generation! Thus, a viable model of a quasar consists of a massive black hole accreting gas from its neighborhood (the reservoir !). The gas, as it spirals in and accelerates becomes hot and emits photons through a variety of radiation processes. The luminosity $L$ may be related to the mass accretion rate $\dot{M}$ as: $L=\kappa \dot{M} c^{2}$, where $\kappa$, an efficiency factor is determined from the nature of the accretion. It is of the order of 0.3 for disc accretion onto a rapidly rotating balck hole (Novikov and Thorne, 1973), where the accreting gas has angular momentum and therefore settles down in the form of a disk around the massive central object.

The mass of the black hole can be estimated from several considerations. For example, one could demand that the gravitational attraction of the accreting gas by the black hole is larger than its repulsion by the emitted radiation. This defines the well known Eddington limit $L_{E}$ and can be derived as: forces due to gravitation and radiation are different for electrons and protons, as a result of which charge separation takes place and an electric field $E$ is set up, the system, then, tries to attain an equilibrium in which the electrons and protons experience equal forces and they 
move together and the luminosity at which this happens is given by $L_{E}=4 \pi G M m_{p} c / \sigma_{T}$, where $G$ is the gravitational constant, $M$ the mass of the black hole, $m_{p}$ the mass of a proton and $\sigma_{T}$ is the Thomson cross-section. One finds $L_{E} \sim 1.3 \times 10^{46} M_{8} \mathrm{erg}$. sec $^{-1}$ where $M_{8} \equiv\left(M / 10^{8} M_{\odot}\right)$. Thus black holes of masses $\sim 10^{8} M_{\odot}$ are needed to power quasars. Further the Schwarzchild radius $R_{s}$ of a black hole is given by $R_{s}=2 G M / c^{2} \sim 3 \times 10^{13} M_{8}$. Thus a characteristic time of variation $t_{\nu}$ which may be associated with a region of this size is $\sim R_{s} / c \simeq 10^{3} M_{8}$ sec. This agrees fairly well with the variability time scale observed in the X-ray part of the spectrum of some sources.

Another way of estimating the mass of the black hole is to attribute the observed width of 5000-10000 $\mathrm{Km} / \mathrm{sec}$ of the emission lines to the Keplerian motion of the gas. One finds that at a distance $R \sim 900 R_{s}$, the Keplerian velocity is of $\sim 10^{4} \mathrm{Km} / \mathrm{sec}$. The absence of broad forbidden lines constrains the electron density in the broad line emission region to $\sim 10^{10} \mathrm{~cm}^{-3}$ and the temperature to a few times $10^{4} \mathrm{~K}$. The observed luminosity in any of the emission lines is much less than would be expected from a region of the size $\sim R$ and further that it cannot exceed the luminosity of a black body at this temperature. This has two fall outs: first, that $R>10^{15} \mathrm{~cm}$ and $M>10^{7} M_{\odot}$ and second that the region of size $R$ is not uniformly filled with gas. It exists in the form of filaments exhibiting several zones of varying density and temperature. Particularly, the two phases with $T \simeq 10^{8} \mathrm{~K}$ and $10^{4} \mathrm{~K}$ have been shown to coexist in pressure and radiative equilibrium (McCray, 1979; Rees, 1984) (for a review see Osterbrock and Mathews, 1986).

The continuum emission of a quasar can be approximated by a power law. The flux $F_{\nu}$ at a frequency $\nu$ goes as $\nu^{-\alpha}$ consisting of several components: In the low frequency $(\nu<1 \mathrm{GHz})$ radio region, $\alpha \simeq 0.1$; in the $\mathrm{X}$-ray region $\alpha \leq 0.7$. The entire spectrum when fitted with an average value of $\alpha \simeq 1$ shows bend in the radio, bumps in the blue and distinct variations in the hard X-ray and $\gamma$-ray regions. The continuum originates very near the black hole and then interacts with the surrounding gas, which, as a result, exhibits phases of diverse temperatures and densities.

The radio emission associated with quasars typically has two distinct components: a compact source coincident with the optical emission showing a flat or complex spectrum and, extended roughly symmetrical double radio sources on both sides of the optical object with a spectral index $\sim 0.7$. This extended emission can exist out to hundreds of Kiloparsec and is usually seen as two sided lobes, though only one of the jets feeding the lobes is usually visible. How the central object feeds and maintains these huge jets has remained an issue of active consideration.

\section{Early models of continuum emission}

The core radio radiation from AGN is believed to be produced via synchrotron process in which relativistic electrons gyrate in an ambient magnetic field. The synchrotron radiation mechanism has been discussed in various astrophysical contexts and the characteristics of a radiation so produced are well documented (Pacholczyk, 1970, Ginzburg and Syrovatssky, 1974; Rybicki and Lightman, 1979). For a power law energy distribution of electrons, synchrotron radiation flux also has a power law spectral distribution with $\alpha=(\beta-1) / 2$ where $\beta$ is the energy index $\left(f(E) \propto E^{-\beta}\right)$ of the electrons. It is found that many of the acceleration processes such as shock acceleration, acceleration by magnetohydrodynamic waves etc. produce a value of $\beta \sim$ 3 . This, then gives a value of $\alpha$ which is close to the observed spectral index to a low frequency turn over. At this turning point, the electrons begin to absorb the radiation they emit and the source is said to be selfabsorbed. The radio flux varies as $f_{\nu}\left(\propto \nu^{5 / 2}\right)$ for frequencies below the turning point. Now this part of the spectrum has not been observed, instead a flat spectrum with $\alpha \sim 0.1-0.3$ is what is often observed. This is explained by invoking the inhomogeneous nature of the radio source, wherein a tailored superposition of $\nu^{5 / 2}$ spectra gives rise to a flat spectrum.

The self-absorption occurs when $T_{B} \sim$ $\left(\gamma / K_{B}\right) m_{e} c^{2} \sim 10^{10} \gamma$, where $\gamma$ is the Lorentz factor of the synchrotron emitting electrons, $K_{B}$ is the Boltzmann constant and $m_{e}$ is the electron mass. $T_{B}$ the brightness temperature at a frequency $\nu$ is defined as: $K_{B} T_{B}=c^{2} F_{\nu} / 2 \pi \theta^{2} \nu^{3}$ where $F_{\nu}$ is the radiative flux and $\theta$ is the angular size of the source. From the observed values of $F_{\nu}, \nu$ and $\theta$ at the turnover frequency, one can determine the Lorentz factor $\gamma$ of the electrons emitting at the turnover frequency. Since the synchrotron turnover occurs at a frequency $\nu=\gamma^{2} 0.3 e B / 2 \pi m c /(1+z)$ one can determine the magnetic field $B$ for a known value of the redshift $z$ and then the total number of relativistic electrons required to account for the observed value of $F_{\nu}$. Thus from the self-absorbed feature of the spectrum, typical values of the physical parameters of the radio emitting plasma can be estimated. The variability aspect of the radio emission will be discussed in a later section.

Linear polarization as high as $75 \%$ is predicted for the synchrotron mechanism and high linear polarization is often seen in extended radio sources. The compact radio sources, however show polarization of a few percent. Thus, depolarization processes are usually supposed to be operative. With faith in the synchrotron mechanism, thus restored, it was proposed 
that at least in radio-loud objects, radio to ultraviolet (and sometimes soft $\mathrm{X}$-ray) radiation is produced by synchrotron process and $\mathrm{X}$-rays to gamma rays are produced through the inverse compton scattering of the synchrotron produced UV photons (Hoyle, Burbidge and Sergent, 1966). The power $P_{s y}$ emitted per electron by the synchrotron mechanism is given by (Rybicki and Lightman, 1979).

$$
P_{s y}=2 / 3\left(c \sigma_{T} \gamma^{2} \beta^{2} B^{2}\right),
$$

where $\sigma_{T}$ is the Thomson cross-section, $\beta c$ is the perpendicular velocity of the relativistic electron with $\gamma$ the Lorentz factor perpendicular to the direction of $\mathrm{B}$, the magnetic field. The power $P_{c}$ emitted per electron by the inverse compton process is given by (Rybicki and Lightman 1979):

$$
P_{c}=2 / 3\left(c \sigma_{T} \gamma^{2} \beta^{2} 4 \pi U_{r a d}\right) \text {, }
$$

where $U_{\text {rad }}$ is the energy density of the synchrotron generated photons.

It was argued by Holye, Burbidge and Sergeant (1966) that the ratio $P_{c} / P_{s y}$ turns out to be much larger than one, infact $\sim 10^{5}$ at a frequency $\nu=10^{14}$ $\mathrm{Hz}$ for $3 \mathrm{C} 273$ and similarly for other objects. This implied that the compton process generated much more power by using synchrotron photons and electrons than did the synchrotron process, leading to a divergence in which the inverse compton process acts on itself to produce higher power at higher and higher frequencies. This dilemma is known as the Compton catastrophe. Jones et al. (1974), however, have criticized this conclusion, for according to them spectral power instead of the total power should be compared since the former is what the observations provide. Further, the relative contribution of synchrotron self-Compton (SSC) can be reduced for anisotropic distribution of relativistic electrons in ordered magnetic fields. Another way out of the compton catastrophe is to assume different values of the Lorenztz factor for producing radiation at different frequencies.

If $L$ is the luminosity and $\mathrm{d}$ the size of the source, $L=\pi d^{2} c U_{\text {rad }}=4 \pi R^{2} F$, where $F$ is the flux received at the earth from the object at a distance $R$. Thus $U_{\text {rad }}=4 F / \theta^{2} c=8 \pi\left(\nu^{3} / c^{3}\right) K_{B} T_{B}<B^{2} / 4 \pi$ gives an upper limit for the brightness temperature $T_{B} \sim 10^{12}-10^{13} \mathrm{~K}$. Detection of rapid variability ( small $\theta$ ) in some sources implies, then, that the brightness temperatures, in these sources, can exceed the SSC limit by several order of magnitude. This difficulty has been resolved by suggesting that the source is expanding relativistically toward the observer (Rees, 1966). The timescale for variability in the source rest frame then increases by the Kinematic Doppler factor $\delta \equiv\left[\gamma_{o}\left(1-\beta_{\| o}\right)(1+z)\right]^{-1}$ and so does the angular size of the source, which results in the reduction of the brightness temperature. Thus relativistic beaming has been found handy for explaining the observed superluminal expansion, the low frequency variability and rapid changes in polarization and intensity in addition to providing a cure for the Compton catastrophe.

Thus the scenario that is popular for the radio-loud and other related objects consists of a jet like structure, emanating from the core. The radiation from radio to UV and sometimes even including $\mathrm{X}$-rays is produced through incoherent nonthermal synchrotron process. Time variability studies provide an estimate of the relative sizes of the emission regions at various frequencies. In some other sources X-rays may be generated by the inverse Compton scattering of low frequency photons. If this process operates then one expects to see correlated variability in the seed photons at infrared and millimeter wavelengths and the Compton scattered photons at X-rays. The overall spectrum has a spectral index close to unity with a very broad peak in the millimeter to ultraviolet region. This feature has a large range in wavelength and probably reflects the range in the parameters of the radiating plasma. Typical values of the parameters may lie in the range: $B \sim 10^{2}-10^{4}$ $\mathrm{G}$, relativistic electron density $n \sim 10^{8}-10^{10} \mathrm{~cm}^{-3}$, Lorentz factor $\gamma \sim 10^{3}-10^{4}$ and the size of the emission region $d \sim 10^{15}-10^{17} \mathrm{~cm}$. This plasma can provide a luminosity $L \sim 10^{44}-10^{46} \mathrm{erg} / \mathrm{sec}$. The Compton limited luminosity is also of the same order. Recent observations from Compton Gamma Ray observatory have revealed a surprising correlation between emission at $\mathrm{Gev}$ and radio frequencies. This lends credence to the inverse Compton scattering process for the generation of gamma-rays (Marscher and Bloom, 1992). The observed Gev energies of $\sim 10^{48} \mathrm{erg} / \mathrm{sec}$ are perhaps the result of relativistic beaming. Time variability of the order of a few days has been seen at gamma-rays, also typical of the optical and UV region, again pointing to the role of upscattering processes (Kniffen et al, 1993).

None of the radio-quiet objects have been seen to emit $\mathrm{GeV}$ photons. As mentioned already, the radio quiet objects have very weak emission of the order of a few milli Jansky at frequencies $\cong 10^{10} \mathrm{~Hz}$ and the emission rises thousand fold to the level of Jansky at far infrared wavelengths. This steep fall with an average slope of 3.75 (Hughes et al., 1985) towards low frequency cannot be accounted by synchrotron self absorption mechanism which can, at best produce a fall with a slope of 2.5. In addition a new component in the form of a bump, between 2 - $10 \mu \mathrm{m}$ lying above the underlying power law has been identified (Robson et al., 1986; Edelson and Malkan, 1986). It has been suggested that hot dust may be responsible for most of the infrared emission in radio-quiet objects (Rees et al., 1969; Rieke, 1978 and Barvainis, 1987). 
Dust is thus assumed to be present in sources displaying the infrared bump. It absorbs the optical ultraviolet radiation from the central continuum and reemits at infrared wavelengths. The power $P_{\nu}$ per unit frequency emitted by a single grain at a temperature $T_{g}$ is given by (Barvainis, 1987).

$$
P_{\nu}=4 \pi a^{2} \pi Q_{\nu} B_{\nu}\left(T_{g}\right) \text { erg } \sec ^{-1} \mathrm{~Hz}^{-1},
$$

where $a$ is the size of a grain and is believed to be of the order of $0.05 \mu \mathrm{m}, Q_{\nu}$ is the absorption efficiency of the grains in the infrared and $B_{\nu}\left(T_{g}\right)$ is the Planck function. A grain exposed to UV radiation will achieve equilibrium when the rate of absorption in the UV equals its rate of emission in the infrared i.e.

$$
\pi a^{2} \int U_{\nu} c Q_{\nu} d \nu=\int P_{\nu} d \nu
$$

where $\left(Q_{\nu} \pi a^{2}\right)$ is the absorption crosssection of a grain and $U_{\nu}$ is the energy density in the ultraviolet. This equality determines the temperature of a grain. One can also determine the distance $\xi$ from the central source at which the temperature $T_{g} \leq 1500 \mathrm{~K}$, which is the evaporation temperature of graphite grains. The optically thin radiation from graphite grains at $T_{g} \geq$ $1500 \mathrm{~K}$ peaks at $\sim 2 \mu \mathrm{m}$ and is suggestive of the infrared bump. The size of the dust region can be determined by acknowledging that at large distance from the UV source, the dust does not absorb enough UV and remains cold and nonradiating in the infrared. Thus the infrared emission is supposed to originate in a region of $\cong 10 \mathrm{pc}$ extent situated about a pc from the central UV source. Predominantly graphite grains of $0.05 \mu \mathrm{m}$ size, either distributed spherically symmetrically or in clumps with a total mass $\sim 10^{3}-10^{5} M_{\odot}$ at a range of temperatures can account for the infrared characteristics of a majority of the radio-quiet objects. It is interesting to recall that the flat radio spectrum was produced by assuming that the synchrotron selfabsorbed source is made up of several different components and here, the steep infrared spectrum is produced by assuming that the emission region consists of dust at many different temperatures? Are their other interpretations?

Advancing towards high frequencies next, comes the big blue bump spread over from $1 \mu \mathrm{m}-0.5 \mathrm{KeV}$ region. It is believed that this spectral region consists of two components, a nonthermal power law radiation with a spectral index $\alpha \simeq 1$ over which lies superimposed thermal emission with a black body spectrum modified due to the spatial variation of the temperature of the emitting region. The fux $F_{a}$ released per unit area from the matter accreted at a rate $\dot{M}$ on to a black hole of mass $M_{H}$ is given by (Shields, 1978).

$$
F_{a} \approx 3 G M_{H} \dot{M} / 8 \pi r^{3}
$$

If most of this power is radiated as if from a black body then the corresponding black body temperature $T_{a}$ is found to be

$$
T_{a}(r)=\left(F_{a} / \sigma\right)^{1 / 4},
$$

where $\sigma=5.67 \times 10^{-5} \mathrm{erg} \mathrm{sec}^{-1} \mathrm{~cm}^{-2} \mathrm{~K}^{-4}$. The frequency of maximum emission at a radius $\mathrm{r}$ can be determined from:

$$
\nu_{\max }(r)=2.8 K_{B} T_{a}(r) / h .
$$

Integrating $T_{a}$ over the area, taking $r_{\min }$ as the inner radius of the accretion disk, one finds energy emitted per sec per $\mathrm{Hz}, f$, to be:

$$
f \sim 1 / \nu_{\max }\left(r_{\min }\right) \int_{r_{\text {min }}}^{\infty} F_{a} r d r=\left[3 G M_{H} \dot{M} \sigma^{1 / 2} / 8 \pi\right]^{2 / 3}\left(2.8 K_{B} / h\right)^{-4 / 3} \nu_{\text {max }}^{1 / 3}\left(r_{\text {min }}\right)
$$

Thus the flux $f$ has a spectral index $\alpha=-1 / 3$. Of course at each spatial point the gas emits a Planckian and the $(1 / 3)$ spectrum results from the spatial variation of the temperature and therefore of the frequency of the maximum emission. For frequencies larger than $\nu_{\max }$ the thermal emission falls off exponentially. This, in essence, is the thermal accretion model of the big blue bump, which lies over the nonthermal continuum extending from far infrared to X-rays. Several variants of this model have been proposed including contributions from dust, but it appears that if the near infrared emission is nonthermal and is due to dust emission, it may be difficult to save the accretion model (Malkan,
1991, 1992; Czerny, 1994).

\section{Alternative - coherent radi- ation processes}

The extremely rapid variability requiring bulk relativistic beaming Lorentz factors of 1000 or larger not exhibited by the observed superluminal expansion, a rather contrived tailoring of the frequency spectrum in order to fit the observations, the need to heat the emission line region to get highly ionized metallic atoms are some of the compelling reasons to look for alternative mecha- 
nisms of radiation generation, propagation and absorption. The Coherent radiative processes can alleviate some of these caveats. And plasma processes offer a variety of ways to generate coherent radiation and heat plasmas to high temperatures. The existence of a variety of time scales associated with different wave modes in a plasma can account for the time variability of the radiation. Most of all the inferred physical parameters in AGN are such that they satisfy the conditions for the occurrence of the plasma processes. The inclusion of these processes in the modeling of AGN phenomena, in return, provides us with several good diagnostics.

\section{Novelties of a nonlinear plasma}

In a nonlinear plasma, the densities, the temperatures and the transport parameters such as the electrical conductivity become functions of the imposed high intensity electromagnetic or electrostatic field. For example high intensity radiation $E_{o}\left(\omega_{o}\right)$ passing through a plasma induces an oscillatory motion $\left(u\left(\omega_{o}\right)\right)$ of the plasma particles which combined with the density fluctuations $\delta n(\omega)$ produce electric current density $J(\omega \pm$ $\left.\omega_{o}\right)$ at the side bands $\left(\omega \pm \omega_{0}\right)$. This gives rise to the electric field $E_{s}\left(\omega \pm \omega_{o}\right)$ which couples with the incident field $E_{o}\left(\omega_{o}\right)$ to reinforce the density fluctuations $\delta n(\omega)$ and the feed back process continues as shown in the Fig. 1. These nonlinear processes affect the propagation, absorption and scattering of radiation and need to be determined self-consistently. These so called stimulated processes can reduce or enhance the plasma transparency depending upon the plasma parameters and the incident radiation.

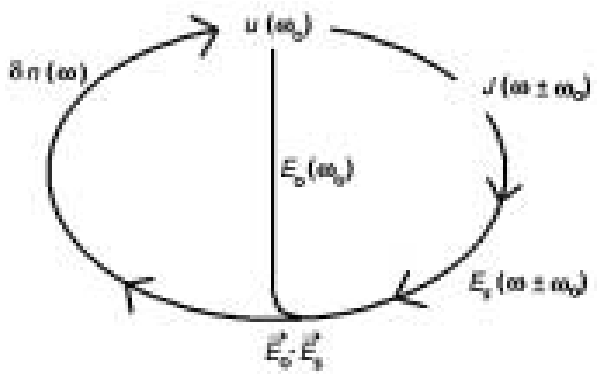

Figure 1. Feed back mechanism for nonlinear plasma instability.

In the next sections, we discuss the role of some of these processes in the generation of the nonthermal continuum of AGN, heating of the emission line region, absorption of the $21 \mathrm{~cm}$ radiation, the variability of the continuum and the electron-positron pair production and annihilation processes (Krishan, 1997).

\section{Stimulated Raman and Compton scattering in quasars}

There are three ways by which the scattering of radiation in a plasma can occur: (1) stimulated Raman scattering (SRS), where a strong electromagnetic wave scatteres off a weakly damped electron plasma wave $\left(K_{e} \lambda_{D e} \ll 1\right)$, (ii) stimulated Compton scattering (SCS), where a strong electromagnetic wave is scattered off a highly damped electron plasma wave $\left(K_{e} \lambda_{D e} \geq 1\right)$ and (iii) the Compton or the Thomson scattering (CS), where the electromagnetic radiation is scattered by single electrons (Fig. 2). Although the stimulated scattering processes are well known among laboratory plasma physicists, they remained unknown to astrophysicists until the work of Krishan (1983). Under appropriate conditions, these plasma processes play a decisive role in the generation as well as the reprocessing of electromagnetic radiation.

\section{COMPTON SCATTERING(INVERSE)}

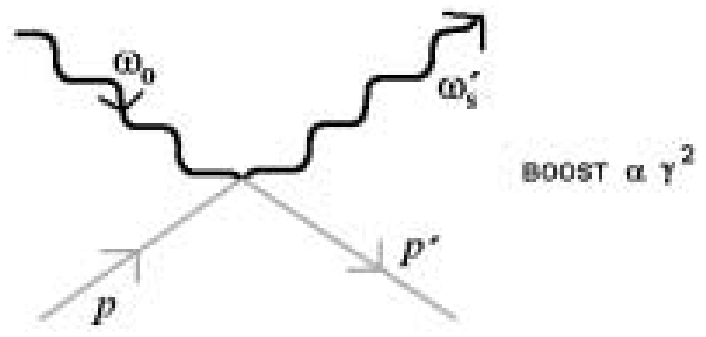

STIMULATED RAMAN SCATTERING

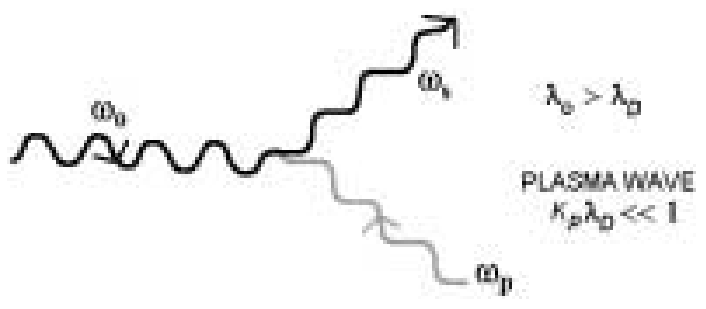

STIMULATED COMPTON SCATTERING

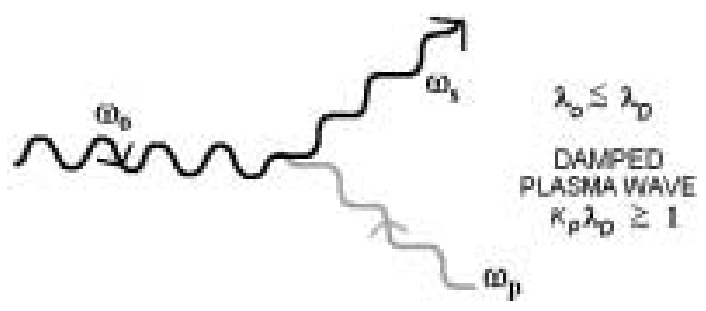

Figure 2. Scattering of radiation in a plasma. 


\section{V.1 Non thermal continuum}

A combination of the stimulated Raman and Compton processes has been proposed to model the entire continuum radiation. In this picture, the gist of which was first proposed by Krishan $(1983,1985)$, a region of suitably varying density containing fast particles and electromagnetic fields can generate the observed continuum all the way from Radio to X-rays (and even $\gamma$ rays). The electrodynamic processes allow particles to be in a state of continuous acceleration as they can be accelerated by the fields they emit (Pacini and Salvati, 1982).

\section{V.2 Energetic electrons}

The electrons are accelerated by electron-plasma waves, which are produced through Raman forwardscattering (RFS). RFS is characterized by the beating of two electromagnetic waves of frequency and wave vector $\left(\omega_{o}, \vec{K}_{o}\right)$ and $\left(\omega_{1}, \vec{K}_{1}\right)$ respectively, so that

$$
\omega_{o}-\omega_{1}=\omega_{e} \text { and } \quad \vec{K}_{o}-\vec{K}_{1}=\vec{K}_{e}
$$

and an electron plasma wave $\left(\omega_{e}, \vec{K}_{e}\right)$ is produced. The two electromagnetic waves, in the context of AGN could possibly arise from cyclotron emission. For $\omega_{o} \gg \omega_{p e}$, the phase velocity of the Langmuir waves $V_{p}=\omega_{e} / K_{e} \cong c\left(1-\omega_{p e}^{2} / \omega_{o}^{2}\right)^{1 / 2}$ is close to $c$. Such a wave will grow in strength until its amplitude $E_{e}$ becomes relativistic; in other words, the quiver velocity of the electron $V_{E}=e E_{e} / m_{e} \omega_{e} c$ approaches $c$. Then the wave traps electrons in the tail of the distribution and accelerates them. Because $V_{p} \cong c$, a sizeable fraction of the electrons will stay in phase with the wave for extended periods and can thus be accelerated to high values of Lorentz factor $\gamma$ (Krishan and Wiita, 1990). A critical account of several popular acceleration processes in astrophysical situations is given in Colgate (1994).

\section{V.3 The scattering agency}

The energetic electrons can radiate by being scattered off either in the Coulomb field of ions (Bremsstrahlung); in a static magnetic field (Synchrotron), in a spatially periodic magnetic field, electrostatic wave and or electromagnetic wave through Raman and Compton processes. The high amplitude Langmuir waves produced by RFS may be susceptible to magnetic modulational instabilities (Bel'kov and Tsytovich, 1979, 1982; Kono, Skoric and ter Haar, 1981). The plasma oscillations with inhomogeneous phase distributions produce vortical currents which tend to increase the spontaneously produced magnetic fields. The magnetic modulational instability excites magnetic fields with spatial periods peaking at $\left(c / \omega_{p e}\right.$, but ranging up to $\left(V_{e} / \omega_{p e}\right)$ with an amplitude $B_{M M}=$ $\left.e E_{e}^{2} /\left(4 m_{e} c \omega_{p e}\right)\right)$. This quasi-stationary spatially periodic magnetic field will remain excited as long as the Langmuir electric field continues to be reinforced via the beating of the two electromagnetic waves. Intense localized regions of electrostatic field in the form of soliton like structures could also provide a scattering agency (Weatherall and Benford, 1991; Lesch and Pohl, 1992). The low frequency electromagnetic waves, say produced by synchrotron processes could also scatter off the energetic electrons via stimulated Raman scattering in addition to the much discussed Compton scattering.

\section{V.4 Production of radiation}

The role of stimulated scattering processes in the generation of AGN nonthermal continuum and its fast variability is being investigated since it was first pointed out (Krishan, 1983, 1985; Krishan and Wiita 1990; Gangadhara and Krishan, 1992; Weatheerall and Benford, 1991; Coppi, Blandford and Rees, 1993; Levinson and Blandford, 1995). The model consists of an electron beam which propagates radially outwards and interacts with the soft photon field to produce radiation at high frequencies. The generation (Blandford and Payne, 1982; Wiita, Kapahi and Saikia, 1982) and stability of extremely sharp electron beams in quasars has been discussed by Lesch and Schlickeiser (1988). The scattering can be studied more easily in the rest frame of the electrons, as is done in the study of inverse Compton scattering. In the rest frame of the electron beam, non-relativistic calculations of the energy transfer rates or the growth rates and the scattered flux can be carried out and these quantities can then be transferred back to the laboratory frame.

The density of beam electrons can be estimated by demanding that the rate at which the beam electrons lose energy from SRS, equals the rate at which they gain energy from RFS. Note that, while an ambient plasma has an important role in the acceleration, only the relativistic beam particles are responsible for the emitted radiation. Further, this ansatz has the pleasant consequences that a steady state supply of relativistic electrons with small thermal spread is available.

The total power emitted by SRS can be estimated by noting that, as the scattered power rises, the effective electric field $\left(\overrightarrow{\beta_{i}} \times \overrightarrow{B_{s}}\right)$ produced by the scattering also increases. This field will trap the beam electrons, increasing their thermal spread and halting the process.

Since, the frequency of emission scales with the electron plasma frequency and therefore the electron density $n$, high frequency emission originates in the denser regions, presumably close to the very core of the AGN. It is interesting to note that the power can be made independent of frequency (Corresponding to $\alpha=1$, close to what is observed in the infrared $\mathrm{X}$-ray region) if, $\gamma$, 
and the product $n A$ are constants. If the beams have a constant opening angle so that $A \propto r^{2}$, then this special case corresponds to $n \propto r^{-2}$, the familiar law of density variation in a uniformly expanding or contracting medium. In general, assuming a power law spatial variations of $n$, frequency $\omega_{s}$ and luminosity $L(r)$ etc. It was shown in Krishan and Wiita (1990), fairly good fits to the average AGN and Seyferts spectra could be obtained for very reasonable values of the various parameters except that electron density is taken to be higher than what is usually assumed.

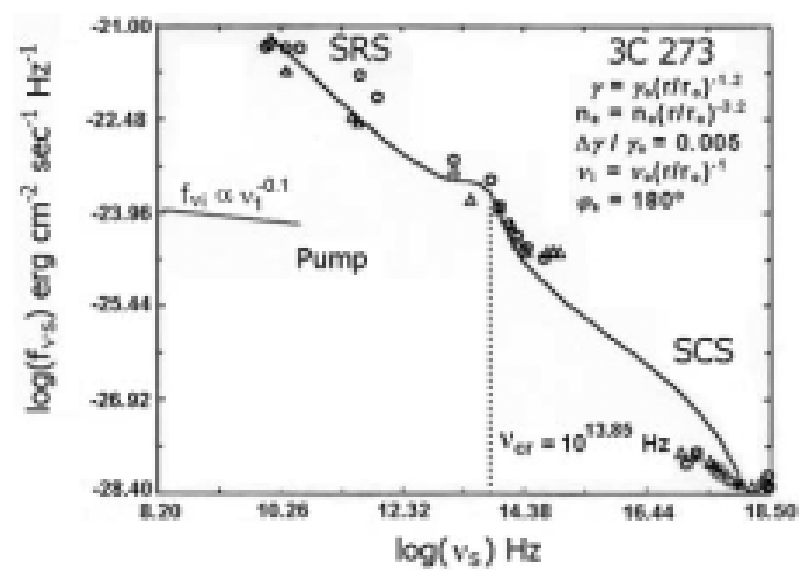

Figure 3. The spectrum of the quasar $3 \mathrm{C} 273$. The solid lines represent the calculated spectrum and the specrum of the pump wave. The observed points, o (1984 February) and $\triangle(1986$ February) by Courvoisier et al. (1987), are also plotted. The constants are: $\gamma_{o}=3 \times 10^{3}, n_{o}=9.24 \times 10^{17}$ $\mathrm{cm}^{-3}, \nu_{o}=4 \times 10^{10} \mathrm{~Hz}$.

The stimulated scattering process for the entire range of plasma and radiation parameters so as to include SRS as well as SCS and hence going through the transition region was studied by Gangadhara and Krishan (1992). Assuming a flat spectrum radio pump, power law spatial variations of plasma density, Lorentz factor, temperature and spectrum of the pump, almost the entire spectrum of $3 \mathrm{C} 273$ was reproduced (Fig. 3). At the critical frequency $\left(\nu_{s} \leq 10^{13.85} \mathrm{~Hz}\right)$ there is a break in the spectrum due to the change of scattering process from SRS to SCS. The SRS process contributes in the lower frequency part $\left(\nu_{s} \leq 10^{13.85} \mathrm{~Hz}\right)$ while SCS contributes in the higher energy part. The slope of the spectrum in the SRS region is $\sim-0.8$ and in the SCS region, it is (-0.7). The hard x-ray part of the spectrum is steep due to strong collisional damping of the scattered and the electron plasma waves and the slope of this part is $\sim(1.5)$. The bump in the spectrum is produced at the transition region $\left(K_{b} \lambda_{D e}=0.4\right)$ between SRS and SCS regions. It is clear that plasma scattering processes can contribute, in a major way, to the production of nonthermal continuum of AGN, along with its polarization and variability characteristics.

\section{Heating of the emission line region}

The strong nonthermal radio continuum from the AGN can heat the emission line region through the action of the parametric decay instability. In this process, the radio wave decays into an electron-plasma wave and an ion-acoustic wave which are then absorbed by the plasma. It is found that temperature of more than $100,000 \mathrm{~K}$ can be attained through this process (Krishan, 1987).

\section{Absorption of the $21 \mathrm{~cm}$ ra- diation}

The absorption of $21 \mathrm{~cm}$ radiation from AGN is generally attributed to the neutral hydrogen clouds. But it is shown that much larger absorption can take place in the ionized regions though the parametric decay instability. The inclusion of this absorption process commands a reestimate of the neutral hydrogen component for the absorption of the $21 \mathrm{~cm}$ radiation (Krishan, 1988).

\section{Continuum variability}

The AGN continuum shows variability on different time scales in the different spectral regions. A host of plasma and MHD waves can introduce quasi-periodic or aperiodic time variability in the emitted radiation. Especially the change of emission process from the Compton to Raman scattering can manifest itself in terms of extremely rapid variability. Further, the inverse Compton scattering by hard electrons could compete with the Langmuir wave emission from them and thereby causing variability in the radio region instead of the X-ray region (Krishan and Wiita, 1994. Krishan, Wiita and Ramadurai, 2000).

\section{Stimulated pair processes}

The production and annihilation of the electron positron pairs play an important role in the $\gamma$-ray region of the AGN radiation. In addition to the Compton process in which two photons and an electron-positron pair participate, the plasma process in which one of the photons has been replaced by an electron-plasma wave can contribute to the pair processes in a significant way. The cross sections are modified in an important quantitative way. All the implications of these processes still remain to be investigated (Krishan, 1999, 2001). 


\section{Conclusion}

The role of nonlinear fast plasma processes in the energetic environs of AGN and other related objects is being explored. The high brightness temperatures and rapid variability necessitate the inclusion of collective processes in the generation and propagation of electromagnetic radiation. I believe a strong case has been made in favour of the potential carried by plasma processes in revealing the workings of intense sources like quasars. But much remains to be done. The first thing, one should take up is to study these parametric processes in magnetized plasmas and then perhaps also in inhomogeneous plasmas. It is well known that the electric field of an electromagnetic wave swells in the resonance region $\left(\omega_{o} \cong \omega_{p e}\right)$ in an inhomogeneous plasma. The energy stored in this enhanced field could be tapped in the form of energetic particles and radiation. More realistic treatments of nonlinear propagation of broad band radiation needs to be performed. As these plasma processes manifest themselves in the form of instabilities, their saturation mechanisms need to be investigated in order to determine the final equilibrium. An unification of the various radiation mechanisms like Bremsstrahlung, Synchrotron, Inverse Compton and Raman scattering could be achieved if one described these processes in terms of wave-wave scattering, since in a moving frame, the coulomb field and the magnetic field become virtual electromagnetic waves. Or perhaps, there is an entirely novel way of modeling the AGN continuum. This could constitute the investigations of radiation characteristics of a turbulent plasma which is known to support a $K^{-1}$ spectrum of electric field fluctuations ( $K$ is a wave vector). How does this translate in terms of the radiation spectrum? The field of plasma - radiation coupling is rich in scope and should be approached with an open mind towards the myriad possibilities (Krishan, 1993, 1994).

\section{Acknowledgements}

I thank the Brazilian Plysical Society and in particular Prof. Maria Virginia Alves of INPE for inviting me to participate in the meeting on physics of plasma. I thank Dr. F.C.R. Fernandes (INPE) for help with the preparation of this manuscript. Thanks are also due to FAPESP (01/06031-8) for supporting my visit to INPE.

\section{References}

Barvainis, R., Hot Dust And The Near Infrared Bump In the Continuum Spectra of Quasars and Active Galactic Nuclie, Ap. J. 320, 537 (1987).

Belkov, S.A. and Tsytovich, V.N., Modulation Excitation of Magnetic Fields, Soviet Phys. JETP 49(4), 565 (1982).
Belkov, S.A. and Tsytovich, V.N., Modulation NonLinear Generation of the Magnetic Fields in dense plasma, Phys. Scripta 25, 416 (1982).

Blandford, R.D., Payne, P.G., Hydromagnetic Flows from Accretion Discs and the production of Radio Jet, Mon. Not. R. Astr. Soc. 199, 883 (1982).

Colgate, S.A., Acceleration in Astrophysics, Physica Scripta, T52, 96 (1994).

Coppi, R, Blandofrd. R.D. and Rees, M.J., Anisotropic Induced Compton Scattering Constraints on Models of Active Galactic Nuclei, Mon. Not. R. Astron. Sco. 262, 603 (1993).

Courvoisier, T.J.L. et al, The Radio To X-ray Continuum Emission of the Quasar 3C273 and its Temporal Variations, Astron. Astrophys. 176, 209 (1987).

Czerny, B., Emission of Accretion Disks, in IAU Symp. 159: Multiwavelength Continuum emission of AGN, Eds. T.J.L. Courvoisier and A. Belcha, Kluwer Academic Publishers, 261, (1994).

Edelson, R.A. and Malkan, M.A., Spectral Energy Distributions of Active Galactic Nuclei Between 0.1 and 100 Microns, Ap. J. 308, 59 (1986).

Ginzburg, V.L. and Syrovatskii, S.I., Development in The Theory of Synchrotron Radiation and its Reabsorption, Annu. Rev. Astron. Astrophys. 7, 375 (1969).

Gangadhara, R.T. and Krishan V., The role of Compton and Raman Scattering in the Quasar Continuum, Mon. Not. R. Astr. Soc. 256, 111 (1992).

Hasegawa, A., Free Electron Laser, Bell System Tech. J. 57, 3069 (1978).

Hoyle, F., Burbidge, G.R. and Sargent, W.L.W., On the Nature of the Quasi Stellar Sources, Nature 209, 751 (1996).

Hughes, P.A.. Aller, H.D. and Aller, M.F., Polarized Radio Outburst in BL LACERTEA II: The Flux and Polarization of a Piston Driven Shock, Ap. J. 298, 301 (1985).

Jones, T.W., ODell, S.L. and Stein, W.A., Physics of Compact Non-Thermal Sources II: Determination of Physical Parameters, Ap. J. 192, 261 (1974).

Kniffen, D.A. et al. Time Variability in The Gamma-Ray Emission of 3C279, Ap. J. 411, 133 (1993).

Kono, M. Skoric, M.M. and Haar, D., Spontaneous Excitation of Magnetic Fields and Collapse Dynamics In a Langmuir Plasma, J. Plasma Phys. 26, 123 (1981).

Krishan, V. Emission Mechanism of Extragalactic X-ray and Radio Sources, Astrophys. Lett. 23, 133 (1983).

Krishan, V. Acceleration and Radiation Processes Around Active Galactic Nuclei, Astrophys. Sp. Sci. 115, 119 (1985).

Krishan, V. The Extraterrestrial Raman Scattering, Current Science 64, 301 (1993).

Krishan, V. Role of Plasma Processes in Astrophysics, Physica Scripta T52, 118 (1994). 
Krishan, V., Space Science Reviews, 80, 445 (1997). Krishan, V. and Wiita, P.J. Coherent Plasma Processes and The Continuum Emission In Active Galactic Nuclei, Mon. Not. R. Astr. Soc. 246, 597 (1990).

Krishan, V., M.N.R.A.S., 226, 629 (1987).

Krishan, V., M.N.R.A.S., 231, 353 (1988).

Krishan, V. and Wiita, P.J., Ap. J. 423, 172 (1994).

Krishan, V. and Wiita, P.J. and Ramadurai, S., Astron. Astrophy, 356, 373 (2000).

Krishan, V., IAU Symp. 195, 359 (1999).

Lesch, H. and Pohl, M., A Possible Explanation for Intraday Variability in Active Galactic Nuclei, Magnetic Reconnection and Coherent Plasma Emission, A and A, 254, 29 (1992).

Lesch, H. Schlickeiser, R., Stabilization and Consequences of Relativistic Electron Bumps in Extragalactic Radio Sources, Astron. Astrophys. 179, 93 (1987).

Levinson, A. and Blandford, R., Raman Scattering in High-Radio-Brightness Astrophysical Systems: Ap- plication to Active Galactic Nuclei, Mon. Not. R. Astron. Soc. 274, 717 (1995).

Malkan, M.A., Accretion Disk Models for Active Galactic Nuclei, Structure and Emission Properties of Accretion Disks, ed. C. Bertout, S. Collin, J.P.Lasota, J. Tran Thanh Van (Gif Sur Yvette: Ed. Frontiers), 165 (1991).

Malkan, M.A., Examining Alternatives to Accretion Disk Models for the AGN Continuum, in Physics of Active Galactic Nuclei, eds. W.J. Duschl and S.J. Wagner, Springer-Verlag, 109 (1992).

Marscher, A.P. and Bloom, S.D., In Compton Observatory Science Workshop, 346 (1992).

McCry, R., Spherical Accretion onto Supermassive Black holes in Active Galactic Nuclei, Proceedings of a Nato Study Institute held at the Institute of Astronomy, Univ. of Cambridge, eds. C. Hazard and S. Mitton, 227 (1979). 